\title{
HPV-16 infection modifies overall survival of Puerto Rican HNSCC patients
}

\author{
Bianca Rivera-Peña ${ }^{1 *}$ (D) Francisco J. Ruíz-Fullana ${ }^{2 \dagger}$, Germán L. Vélez-Reyes ${ }^{1}$, Rosa J. Rodriguez-Benitez ${ }^{3}$, \\ María J. Marcos-Martínez ${ }^{4,5}$, Juan Trinidad-Pinedo² and Adriana Báez ${ }^{2,6}$
}

\begin{abstract}
Background: HPV-16 modifies the overall survival (OS) of patients with oropharyngeal cancer (OPSCC). HPV-16 has been established as risk factor for OPSCC, but HPV-16 infection may also reside in the larynx and oral cavity. We evaluated HPV-16 status on OS of Head and Neck Squamous Cell Carcinoma (HNSCC) patients.

Methods: HPV-16 infection was confirmed by amplification of E6 and E7 viral oncogenes through PCR assay and E6 IHC in 185 HNSCC samples. Associations between HPV-16 status and clinicopathological parameters were performed using Fisher's exact test and $x^{2}$. Survival analysis was completed using Kaplan-Meier estimator and multivariate Cox regression analysis.

Results: OS of HPV-16 positive patients was longer compared to HPV-16 negative patients $(P=0.002)$. HPV-16 positive tumors of the larynx (LSCC) and pharynx (PSCC) showed improved OS compared to HPV-16 negative tumors. Also, HPV-16 positive patients exposed to radiotherapy presented a better survival.

Conclusions: HPV-16 status has a positive prognostic value in HNSCC. Addition of HPV-16 status to the TNM staging can provide better assessment in prognosis and guide treatment for HNSCC patients.
\end{abstract}

Keywords: Head and neck squamous cell carcinoma, Human Papillomavirus (HPV), Overall Survival (OS), TNM, Biomarker

\section{Background}

HNSCC is the seventh most common type of cancer diagnosed, and it is ranked as the eighth cause of cancer death worldwide $[1,2]$. This cancer includes tumors from the oral cavity (OSCC) (ICD-10-C14.8), pharynx (PSCC) (ICD-10-C14.0), larynx (LSCC) (ICD-10-C32.9), and the paranasal sinuses (ICD-10-C31.9) [3]. HNSCC is predominantly diagnosed in patients over 60 years old; however, a growing number of HNSCC patients are being diagnosed at younger ages [4]. Historically, HNSCC has been more frequently diagnosed in men, with a male-female ratio of about 4:1. However, this ratio is rapidly changing because more women are exposing themselves to tobacco and alcohol [5]. The overall-5year survival (OS) for HNSCC patients is $65.9 \%$, for all HNSCC sites and stages [6], with a median survival of 2.5 years after treatment.

\footnotetext{
* Correspondence: bianca.rivera@upr.edu

${ }^{\dagger}$ Equal contributors

${ }^{1}$ Department of Biology, Natural Sciences Faculty, University of Puerto Rico, Rio Piedras Campus, San Juan, Puerto Rico

Full list of author information is available at the end of the article
}

In the United States, Puerto Rican Hispanics, AfricanAmericans, and economically disadvantaged Whites are at greater risk of developing HNSCC. The incidence of HNSCC in Puerto Ricans is 2.5 higher than Hispanics in the US [7]. The incidence of OSCC or PSCC is approximately $72 \%$ higher in Puerto Ricans than in US Hispanics. Similarly, the incidence rate of LSCC is $51 \%$ higher than among Hispanics living in the Unites States [7].

The etiology of HNSCC involves a variety of toxic, environmental, and viral agents [5]. Studies have established that smoking and alcohol consumption are the major risk factors for the development of HNSCC [8-10]. Currently, human papillomavirus (HPV) infection has also been recognized as a risk factor for HNSCC, particularly for OPSCC [11-13]. There are more than 180 types of HPVs described, of which 30 types are considered high risk, including HPV-16 and HPV-18 [14, 15]. The malignant transformation of HPV integration is mediated by HPV oncoproteins E6 and E7 [14]. HPV-16 E6 protein has been associated to the abnormal degradation of the $\mathrm{p} 53$ protein, leading to a disruption in G1/S cell cycle control [16]. 
Also, HPV-16 E7 oncoprotein binds to the phosphorylated form of $\mathrm{pRb}$ protein, which inactivates $\mathrm{pRb}$ and a disruption in the G1/S transition occurs [17]. Both events cause an abnormal promotion of cell proliferation due to disruptions in the cell cycle control mechanisms. HPV-16 DNA has been detected in almost $35 \%$ of HNSCC patients, and evidence has accumulated showing that HPV is etiologic for OPSCC $[18,19]$. It has been proposed that HPV-16 positive HNSCC patients have a distinct cancer progression and prognosis than HPV-16 negative HNSCC patients [20]. HPV-16 positive patients tend to be diagnosed at a younger age when compared to HPV-16 negative patients [20, 21]. Additionally, the presence of HPV-16 in HNSCC patients has been correlated to the presence of local metastases, positive lymph nodes, and a more advanced tumor stage at the time of diagnosis [22]. Clinically, HPV-16 positive HNSCC patients have a better prognosis than HPV-16 negative patients [11, 13, 23, 24].

The complex anatomical structure of the head and neck area makes it very challenging for clinicians to determine the primary site of HNSCC [25]. Detection of HNSCC involves clinical and histological examinations of suspicious tissue, but, at times, unnoticed malignant lesions remain undetected. HNSCC tumors arising from each anatomical site have a unique progression, epidemiology, and therapeutic approach. HNSCC prognostication is based on the TNM Classification of Malignant Tumors (TNM) according to the sub-site [26]. The TNM system is useful to describe the extent of the disease, estimate the likely prognosis, and plan treatment. Treatment strategies rely on TNM, possible side effects, and the patient's preferences and overall health. Since HNSCC is often discovered in advance stages (III and IV), the most urgent problem is the need to identify an effective diagnostic marker for early detection, and prediction of outcome. Therefore, the purpose of this study was to evaluate whether addition of HPV-16 status to the TNM staging system will help predict better the OS of HNSCC Puerto Rican patients.

\section{Methods}

\section{Study design}

This is a retrospective study where patients meeting the following criteria were eligible for inclusion: histologically proven squamous cell carcinoma arising from the pharynx (hypopharynx, oropharynx), oral cavity, and larynx treated surgically between 1993 and 2005. Fresh-frozen tumor tissue was collected from all HNSCC accrued patients. Additionally, genomic DNA of HNSCC patients had been previously tested for HPV-16 status by Gp5+/6+ primer region within the L1 gene consensus PCR [27], HPV-16 E6/E7 type-specific PCR, and E6 immunohistochemical (IHC) staining [13, 28, 29]. The cohort consisted of 185 HNSCC and their clinicopathological parameters are shown in Table 1. All procedures have the approval of the
Table 1 Study cohort clinicopathological characteristics

\begin{tabular}{|c|c|}
\hline Characteristics & $N=185$ \\
\hline \multicolumn{2}{|l|}{ Age (y) } \\
\hline Mean \pm SD & $62.72 \pm 12.13$ \\
\hline Range & $24-98$ \\
\hline \multicolumn{2}{|l|}{ Sex, n (\%) } \\
\hline Male & $164(88.6)$ \\
\hline Female & $21(11.4)$ \\
\hline \multicolumn{2}{|c|}{ Primary Tumor Site, n (\%) } \\
\hline Larynx & $83(44.9)$ \\
\hline Oral Cavity & $68(36.7)$ \\
\hline Oropharynx & $17(9.2)$ \\
\hline Hypopharynx & $17(9.2)$ \\
\hline \multicolumn{2}{|l|}{ HPV-16 Status, n (\%)* } \\
\hline HPV-16 + & $97(52.4)$ \\
\hline HPV-16 - & $88(47.6)$ \\
\hline \multicolumn{2}{|l|}{ Tumor Stage, n (\%) } \\
\hline I, II & $47(25.4)$ \\
\hline III, IV & $138(74.6)$ \\
\hline \multicolumn{2}{|l|}{ Tumor Grade, n (\%) } \\
\hline Well & $43(23.2)$ \\
\hline Moderate & $107(57.8)$ \\
\hline Poor & $16(8.7)$ \\
\hline SCC & $19(10.3)$ \\
\hline \multicolumn{2}{|l|}{ Nodal Involvement } \\
\hline Yes & $64(34.6)$ \\
\hline No & $121(65.4)$ \\
\hline Heavy Smoking, n (\%) & $163(88.1)$ \\
\hline Heavy Drinking, n (\%) & $154(83.2)$ \\
\hline
\end{tabular}

*HPV-16+ = human papillomavirus type 16 positive; HPV-16 - = human papillomavirus type 16 negative

University of Puerto Rico-Medical Sciences Campus IRB (MSC-IRB Protocol 2770103). Relevant diagnostic information including tumor site, tumor grade, and histology were obtained from medical records and pathological reports. Treatment of choice was surgery followed by postoperative radiotherapy. Follow-up information was prospectively collected from hospital, pathological records and the Puerto Rican Cancer Registry.

\section{DNA extraction}

Genomic DNA from all tumor samples was isolated using the DNA Isolation kit for Cells and Tissues (Roche, Indianapolis, IN) according to the manufacturer instructions. DNA concentration was measured with NanoDrop 8000 UV-vis Spectrophotometer (Thermo Scientific, Waltham, MA). 


\section{Detection of HPV16 DNA}

HPV-16 status had been pre-screened by Gp5+/6+ consensus PCR followed by HPV-16 E6/E7 type-specific PCR, and results were confirmed for this study with a TaqMan-based qPCR targeted at HPV-16 E6 and E7 viral oncogenes. The HPV-16 E6 specific primer set included a forward primer $5^{\prime}$-gcacagagctgcaaacaactataca-3', a reverse primer $5^{\prime}$-tcccgaaaagcaaagtcatatacc- $3{ }^{\prime}$, and a probe oligo $5^{\prime}$-tgtactgcaagcaacagttactgcgacgt- 3 '. The HPV-16 E7 specific primer set included a forward primer $5{ }^{\prime}$-gatgaaatagatggtccagc-3', a reverse primer $5^{\prime}$-gctttgtacgcaaccgaagc-3', and a probe oligo $5^{\prime}$-cggac agagcccattacaatattgtaacc-3'. Quality and amount of input DNA samples were tested in each qPCR assay with $\beta$-actin gene primers with a forward primer $5^{\prime}$-gc ccatctacgaggggta- $3^{\prime}$, a reverse primer $5^{\prime}$-ccttaatgtcacgc acga-3', and a probe oligo 5'-accaccacggccgagcgg- 3 '. Reaction mixtures with SiHa DNA (1-2 copies of HPV-16) and K562 DNA (HPV-16 negative) were used as positive and negative control, respectively. qPCR reactions were carried out in a 96-well optical tray with a final volume of $25 \mu \mathrm{L}$. Each reaction consisted of $600 \mathrm{nM}$ of each primer, $200 \mathrm{nM}$ of each probe (Taqman, Applied Biosystems, Grand Island, NY), 1X of TaqMan Universal PCR Master Mix (Applied Biosystems), which contains the Taq Polymerase, dNTPs, and ROX reference dye, and $75 \mathrm{ng}$ of genomic DNA. DNA was amplified in a 7500 Real Time PCR System (Applied Biosystems, Grand Island, NY). Thermal cycling conditions were: $50{ }^{\circ} \mathrm{C}$ for $2 \mathrm{~min}, 95{ }^{\circ} \mathrm{C}$ for $10 \mathrm{~min}$, followed by 40 cycles of $95{ }^{\circ} \mathrm{C}$ for $15 \mathrm{~s}$ and an annealing temperature of $60^{\circ} \mathrm{C}$ for $1 \mathrm{~min}$. All HNSCC samples classified as HPV-16 positive had amplification of E6 and E7 viral oncogenes through qPCR assay.

\section{Statistical analysis}

Data from independent groups was compared using Fisher exact test or $\mathrm{x}^{2}$, as appropriate. Odds ratio (OR) calculations for clinicopathological parameters were performed using binary logistic. Overall survival (OS) was measured in months from the date of diagnosis until death, if occurred. Survival analyses were performed using Kaplan-Meier curves. Log-rank Mantel-Cox and Gehan-Breslow Wilcoxon tests were used to determine the significance between two survival curves. Established prognostic factors having an impact on HNSCC survival were analyzed in a multivariate Cox regression analysis. Statistical significance was established to be $p \leq 0.05$. Statistical analyses were performed using IBM SPSS Version 22 (IBM Corp; Armonk, NY).

\section{Results}

HPV-16 status in a cohort of HNSCC Puerto Rican patients Patient characteristics are shown in Table 1. The mean age was 62.72 years (range 24-98 SD: 12.13). Of the 185
HNSCC patients included in the study, $88.6 \%$ were male and $11.4 \%$ were female. Three-quarters $(74.6 \%)$ of the patients presented tumors with advance staging (III and IV) and $25.4 \%$ were in early stages (I and II) of the disease. The HNSCC sub-site distribution was $44.9 \%$ LSCC, $36.7 \%$ OSCC and 18.4\% PSCC. The PSCC sub-site includes cases from the oropharynx and hypopharynx. Patients with oropharyngeal and hypopharyngeal cancers were combined under PSCC for the statistical analysis in view of the relatively small number of cases of each subsite in our study cohort. However, the distribution of oropharyngeal and hypopharyngeal cancers is shown in Table 1. Smoking and drinking habits of our study cohort

Table 2 Adjusted OR's and $95 \%$ Cls of HNSCC patients according to HPV-16 status and clinicopathological parameters

\begin{tabular}{|c|c|c|c|c|c|c|}
\hline \multirow[t]{2}{*}{ Variable } & \multicolumn{2}{|c|}{$\begin{array}{l}\text { HPV-16+ } \\
(N=97)\end{array}$} & \multicolumn{2}{|c|}{$\begin{array}{l}\text { HPV-16 - } \\
(N=88)\end{array}$} & \multirow[t]{2}{*}{ OR value, $95 \% \mathrm{Cl}$} & \multirow[t]{2}{*}{$P$ value } \\
\hline & No. & Percent & No. & Percent & & \\
\hline \multicolumn{7}{|l|}{ Age (y) } \\
\hline$\leq 60$ & 45 & 46.4 & 38 & 43.2 & & \\
\hline$>60$ & 52 & 53.6 & 50 & 56.8 & $1.14[0.637-2.035]$ & 0.767 \\
\hline \multicolumn{7}{|l|}{ Sex } \\
\hline Male & 89 & 91.8 & 75 & 85.2 & & \\
\hline Female & 8 & 8.2 & 13 & 14.8 & $1.93[0.759-4.902]$ & 0.173 \\
\hline \multicolumn{7}{|l|}{ Tumor site } \\
\hline Larynx & 41 & 42.3 & 42 & 47.7 & $0.866[0.304-2.470]$ & 0.788 \\
\hline $\begin{array}{l}\text { Oral } \\
\text { Cavity }\end{array}$ & 36 & 37.1 & 32 & 36.4 & $0.998[0.338-2.942]$ & 0.996 \\
\hline Pharynx & 20 & 20.6 & 14 & 15.9 & $1.675[0.420-6.681]$ & 0.465 \\
\hline \multicolumn{7}{|c|}{ Stage of Disease } \\
\hline $1, \|$ & 30 & 30.9 & 17 & 19.3 & & \\
\hline$\| I, I V$ & 67 & 69.1 & 71 & 80.7 & $1.87[0.945-3.700]$ & 0.091 \\
\hline \multicolumn{7}{|l|}{ Tumor grade } \\
\hline SCC & 11 & 11.3 & 8 & 9.1 & & \\
\hline Well & 22 & 22.7 & 21 & 23.9 & $0.875[0.226-3.385]$ & 0.847 \\
\hline Moderate & 55 & 56.7 & 52 & 59.1 & $0.729[0.269-1.973]$ & 0.534 \\
\hline Poor & 9 & 9.3 & 7 & 7.9 & $0.720[0.240-2.161]$ & 0.558 \\
\hline
\end{tabular}

Nodal involvement

\begin{tabular}{|c|c|c|c|c|c|c|}
\hline Yes & 30 & 16.2 & 34 & 18.4 & & \\
\hline No & 67 & 36.2 & 54 & 29.2 & 0.711 [0.387-1.306] & 0.272 \\
\hline \multicolumn{7}{|c|}{ obacco status } \\
\hline Yes & 88 & 90.7 & 75 & 85.2 & & \\
\hline No & 9 & 9.3 & 13 & 14.8 & $1.70[0.686-4.186]$ & 0.265 \\
\hline \multicolumn{7}{|c|}{ Icohol status } \\
\hline Yes & 82 & 84.5 & 72 & 81.8 & & \\
\hline No & 15 & 15.5 & 16 & 18.2 & $1.22[0.561-2.630]$ & 0.695 \\
\hline
\end{tabular}

HPV-16 + human papillomavirus type 16 positive, HPV-16 - human papillomavirus type 16 negative, OR odds ratio, $95 \% \mathrm{Cl} 95 \%$ confidence interval 
were defined according to the substance usage reported by each consented patient. Heavy smoking patients reported smoking a pack or more of cigarettes per day and heavy drinking patients reported 15 drinks or more per week. The majority of our HNSCC patients were heavy smokers (88.1\%) and heavy drinkers (83.2\%).

When we distribute the study cohort by HPV-16 status, $52.4 \%$ were HPV-16 positive and $47.6 \%$ were HPV-16 negative. The HNSCC sub-site distribution of the HPV-16 positive patients was $42.3 \%$ LSCC, $37.1 \%$ were OSCC and $20.6 \%$ were PSCC. No statistically significant association was found between HPV-16 status and gender, age, risk factors and staging (Table 2).

\section{Survival analysis of HNSCC patients according to HPV-16 status}

HNSCC patients with HPV-16 positive status had a better OS compared to HPV-16 negative patients for all sites and stages (Fig. 1). HPV-16 positive patients had a median survival of 102.8 months compared to HPV-16 negative tumors, which had a 63.1 months median survival $(P=0.002)$. Cox regression analysis was performed and showed that HPV-16 positive HNSCC patients have

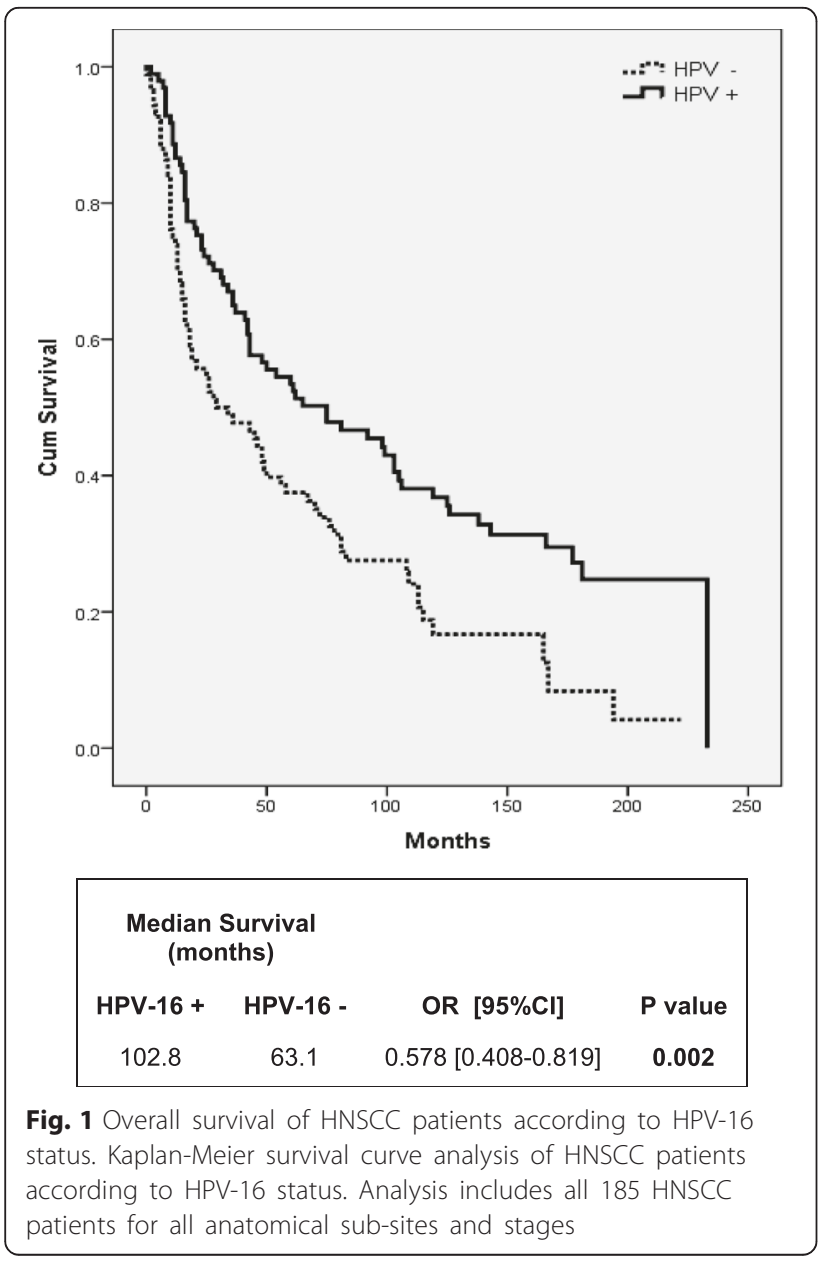

an improved survival compared to HPV-16 negative patients after adjustment for established prognostic factors such as nodal status, tumor stage, cell differentiation, tumor site, heavy smoking and heavy drinking usage, age, and sex (Table 3).

LSCC and PSCC patients with HPV-16 positive tumors had a better OS compared to HPV-16 negative patients (Fig. 2a, 2b). The median survival of HPV-16 positive LSCC patients was 118 months compared to HPV-16 negative cases which had a median survival of 58.1 months $(P=$ $<0.001)$. As well, the OS of PSCC patients improves in HPV-16 positive cases (129.2 months) compared to HPV-16 negative cases $(20.4$ months $)(P=<0.001)$. In contrast, the OS of OSCC patients was not significantly different between HPV-16 positive and HPV-16 negative cases (Fig. 2c).

Since HPV-16 positive patients showed a better survival, we evaluated the effect of HPV-16 presence on OS in HNSCC tumor staging. As shown in Fig. 3, HPV-16 positive patients have a better OS than HPV-16 negative patients regardless of the tumor staging. HPV-16 positive early stage tumors had an improved OS of 106.8 months as compared to HPV-16 negative tumors, which had a median OS of 63.2 months $(P=0.056)$ (Fig. 3a). Likewise, late stage tumors that were HPV-16 positive had a better OS (95.2 months) compared to late stages HPV-16 negative tumors, whose median OS was 60.1 months $(P=0.011)$ (Fig. 3b).

Recent studies have observed that HPV-16 positivity is a strong prognostic marker for OS in patients treated with primary radio-chemotherapy [30]. We compared

Table 3 Multivariate Cox analysis

\begin{tabular}{|c|c|c|c|c|}
\hline Covariate & Coefficient & $\begin{array}{l}\text { Standard } \\
\text { error }\end{array}$ & $P$ value & $\mathrm{HR}$ value, $95 \% \mathrm{Cl}$ \\
\hline $\begin{array}{l}\text { Positive nodal } \\
\text { status }\end{array}$ & 0.308 & 0.209 & 0.141 & $1.361[0.903-2.052]$ \\
\hline Tumor stage & 0.034 & 0.099 & 0.733 & $1.034[0.852-1.257]$ \\
\hline \multicolumn{5}{|l|}{ Tumor grade } \\
\hline Well & 0.089 & 0.442 & 0.841 & $1.093[0.460-2.598]$ \\
\hline Moderate & 0.348 & 0.322 & 0.279 & $1.417[0.754-2.662]$ \\
\hline Poor & 0.496 & 0.344 & 0.149 & $1.642[0.837-3.222]$ \\
\hline \multicolumn{5}{|l|}{ Tumor site } \\
\hline Larynx & -0.170 & 0.337 & 0.614 & $0.844[0.436-1.634]$ \\
\hline Oral Cavity & -0.176 & 0.350 & 0.616 & $0.839[0.423-1.665]$ \\
\hline Pharynx & 0.237 & 0.423 & 0.575 & $1.267[0.554-2.901]$ \\
\hline Heavy smoking & 0.202 & 0.329 & 0.539 & $1.224[0.642-2.333]$ \\
\hline Heavy drinking & 0.217 & 0.249 & 0.384 & $1.242[0.762-2.024]$ \\
\hline HPV-16 & 0.555 & 0.182 & 0.002 & $1.742[1.219-2.488]$ \\
\hline Sex & -0.247 & 0.324 & 0.447 & $0.781[0.414-1.475]$ \\
\hline Age & 0.026 & 0.007 & 0.000 & $1.026[1.011-1.041]$ \\
\hline
\end{tabular}



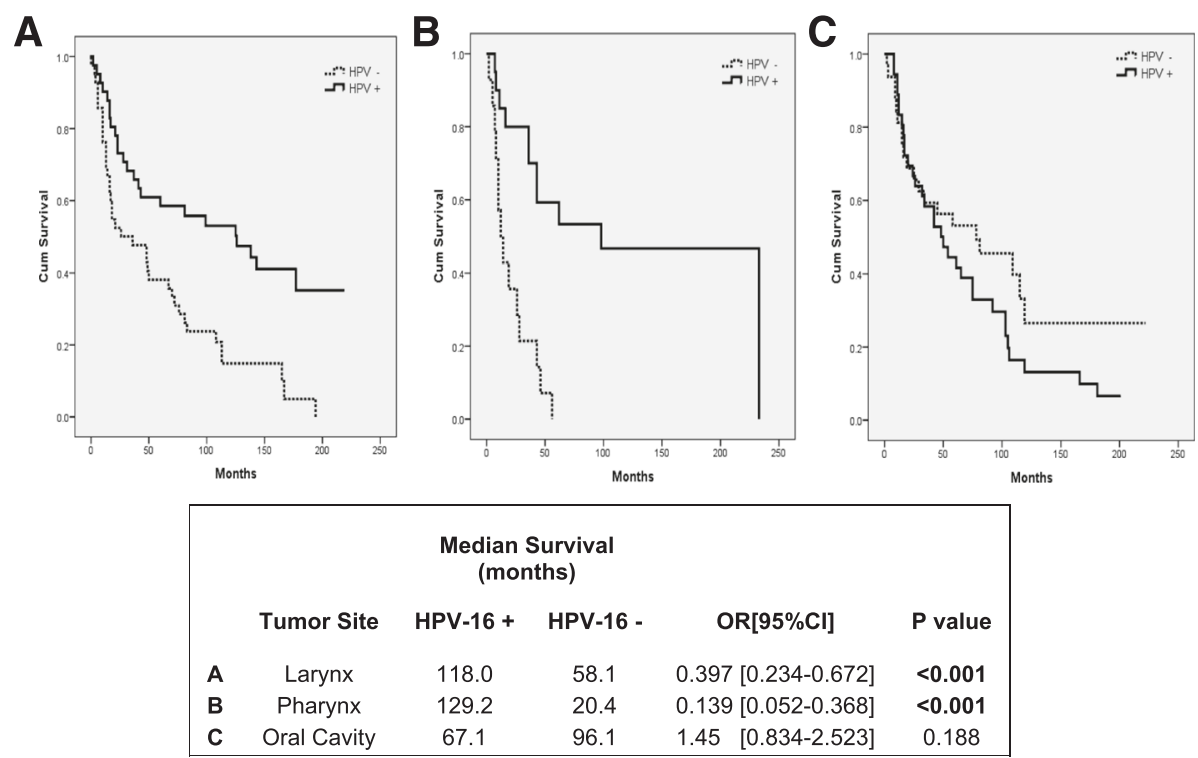

Fig. 2 Overall survival of HNSCC patients according to HPV-16 status in each HNSCC anatomical sub-sites. Kaplan-Meier survival curve analysis of HPV-16 positive patients versus HPV-16 negative patients according to HNSCC anatomical sub-site. a Larynx (LSCC); b Pharynx (PSCC); c Oral Cavity (OSCC)

the OS of HNSCC patients, of all sub-sites and tumor stages, which were treated with radiotherapy adjuvant to surgery. We found that HPV-16 positive patients exposed to radiotherapy after surgery had an improved survival compared to HPV-16 negative patients (Fig. 4a). Furthermore, when we sub-divide HNSCC patients by tumor site and radiotherapy treatment, HPV-16 positive LSCC and PSCC patients showed a better OS than HPV-16 negative patients (Fig. 4b, 4c). In contrast, OSCC patients, treated with adjuvant radiotherapy, do not show difference in OS in the presence or absence of HPV-16 (Fig. 4d).
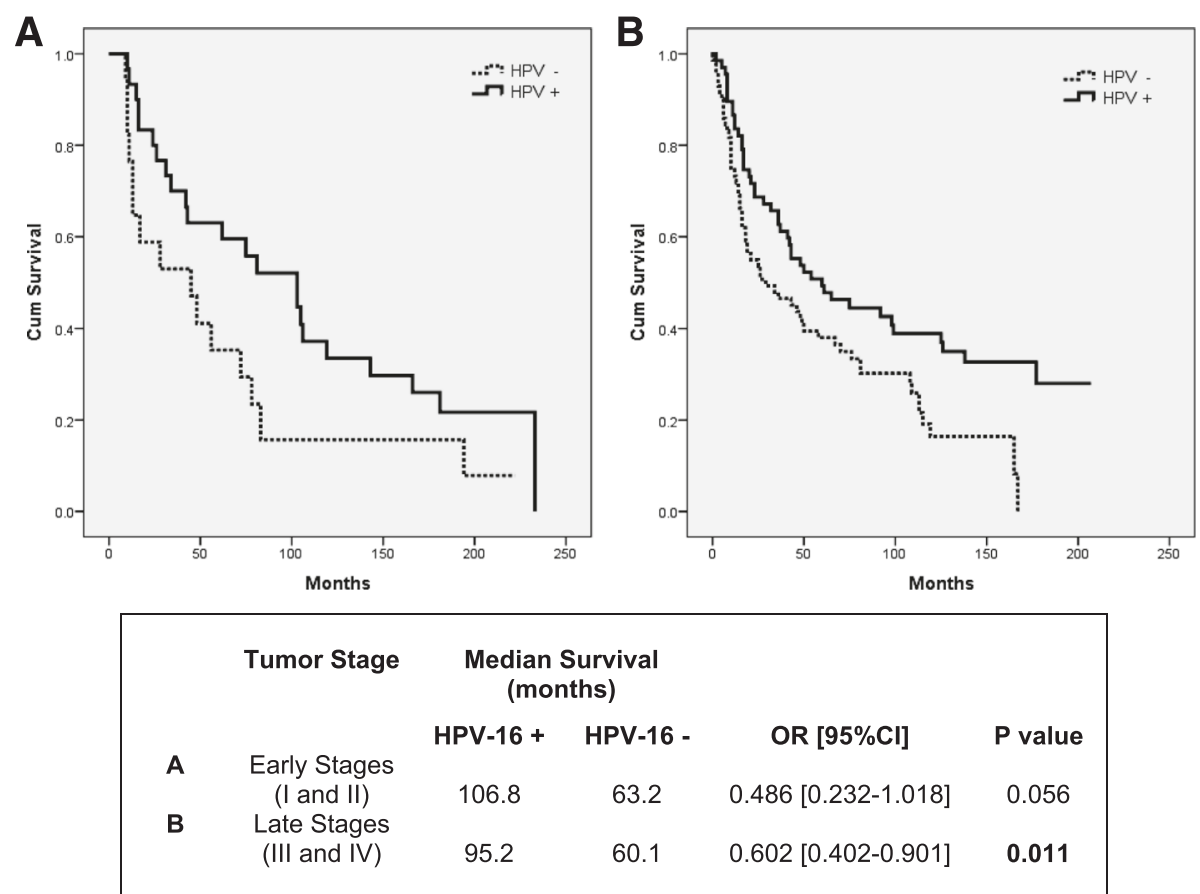

Fig. 3 Overall survival of HNSCC patients according to HPV-16 status and tumor staging. Kaplan-Meier survival curve analysis of HNSCC HPV-16 positive patients versus HPV-16 negative patients distributed by tumor staging. a Early Stages (I and II); b Late Stages (III and IV) 

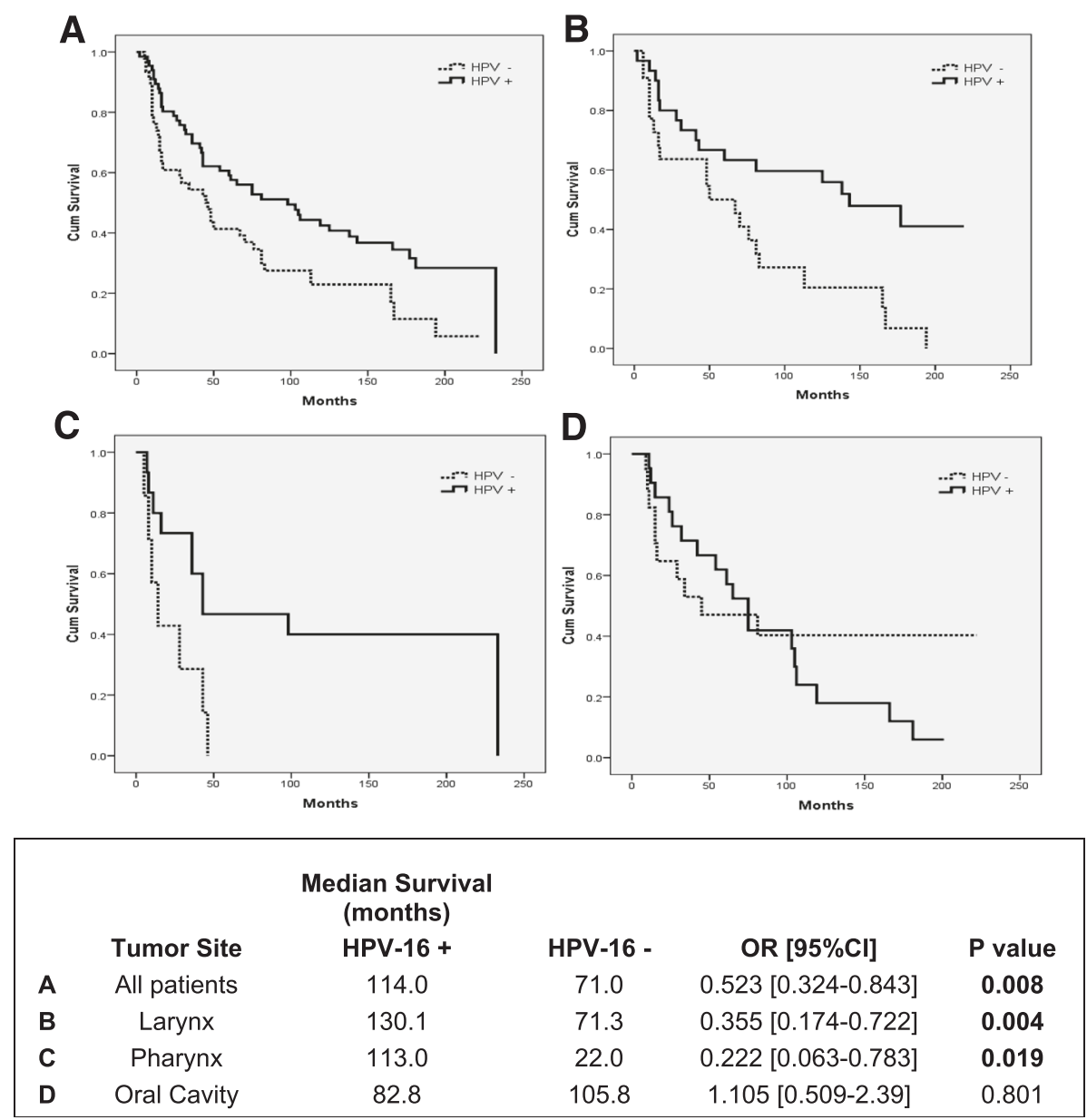

Fig. 4 Overall survival of HNSCC patients exposed to radiation according to HPV-16 status and HNSCC sub-sites. Kaplan-Meier survival curve analysis of HNSCC patients exposed to radiotherapy (RTX) adjuvant to surgery. Patients were distributed by HPV-16 status and anatomical sub-site. a All RTX patients, which includes all HNSCC sub-sites and stages; b Larynx RTX patients; c Pharynx RTX patients; d Oral Cavity RTX patients

Since our study cohort was mostly composed of heavy smokers (88\%), we evaluated if HPV-16 status affects the OS of a HNSCC smoker population. As shown on Fig. 5, HPV-16 positive heavy smokers had a better OS (104.2 months) compared to HPV-16 negative heavy smokers (57.3 months) $(P=<0.001)$. Likewise, the OS of HPV-16 positive patients with a history of heavy alcohol consumption was better (99.9 months) than HPV-16 negative patients (65.1 months) $(P=0.012)$ (Fig. 6).

\section{Discussion}

Our study shows that HPV-16 positivity modifies the OS of HNSCC patients in two anatomical sub-sites, PSCC and LSCC, but not in OSCC (Fig. 2). Previous studies have shown that HPV-16 infection in LSCC and PSCC increased the survival of HNSCC patients [11, 31]. In contrast, a recent study shows that HPV-16 infection in OSCC does not cause an increase in survival, supporting our findings [32].

The key mechanism for which HPV-16 infection gives a better prognosis to HNSCC patients is still unknown.
However, it has been proposed that HPV-16 positive cells have an increased sensitivity to cancer therapies, a slower cellular growth rate, an enhanced immune response towards the virus, or a combination of these factors [33]. Additionally, it has been shown that HPV-16 positive cells have fewer DNA copy number alterations, less genome-wide hypomethylation, less TP53 mutations, and lower expression of EGFR [34]. Because of those differences, two HNSCC carcinogenesis models have being proposed. The first model suggests that progression of HNSCC, not infected by HPV-16, may be due to the amplification or loss of large parts of chromosome arms $3 p, 9 p, 11 p$ and $17 p$ [35-37]. In contrast, the second model of HNSCC carcinogenesis suggests that tumors infected with HPV-16 have a lower level of chromosomal loss at these regions, which may be the cause for a better survival in these patients [37].

It has been proposed that HPV-16 positivity in HNSCC produces distinct tumor sub-site differences when exposure risks are combined, suggesting that 


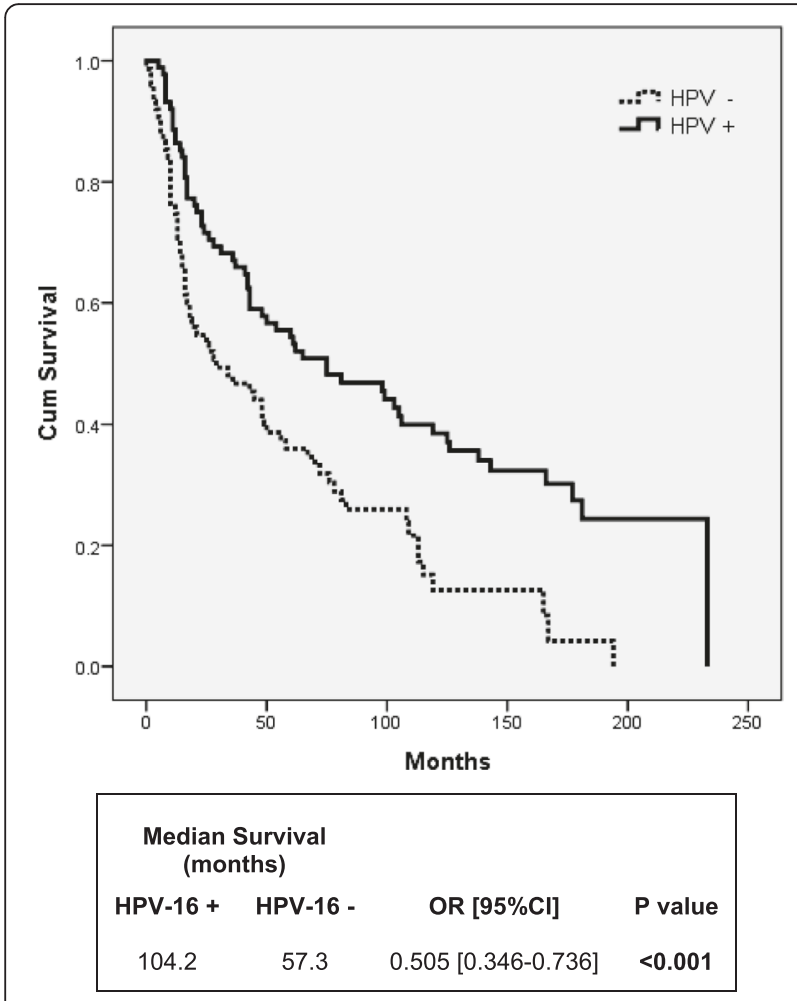

Fig. 5 Overall survival of HNSCC patients with heavy smoking history according to HPV-16 status. Kaplan-Meier survival curve analysis of HPV-16 positive versus HPV-16 negative HNSCC patients with a history of heavy smoking

different molecular pathways are involved [38]. When we evaluated HPV-16 presence in each HNSCC anatomic sub-site, we demonstrated that HPV-16 status has a unique impact in the patient's survival. HPV-16 positive LSCC and PSCC patients have an improved survival, in contrast to OSCC patients which did not show an improved survival. This difference may arise due to smoking and drinking habits of our study population. Our study cohort is composed of heavy smokers $(88.1 \%)$ and heavy drinkers $(83.2 \%)$. It has been proposed that HNSCC carcinogenesis in HPV-16 positive tumors, with a history of heavy smoking, may arise upon HPV-16 infection in pre-neoplastic tissue already having a number of genetic alterations, for instance, $p 53$ mutations or an increase in EGFR expression [39]. If such alterations are acquired prior to HPV-16 infection, it may impart some of the molecular characteristics of HPV-16 negative tumors, thus resulting in poor outcome and prognosis [40]. Also, it has been suggested that tobacco, alcohol and HPV-16 are independent risk factors for HNSCC, producing distinct tumor groups with different prognosis and guide of treatment [41].

HPV-16 status is an important factor for establishing the prognosis and treatment of HNSCC. Our results showed that HPV-16 positive patients had a better response to

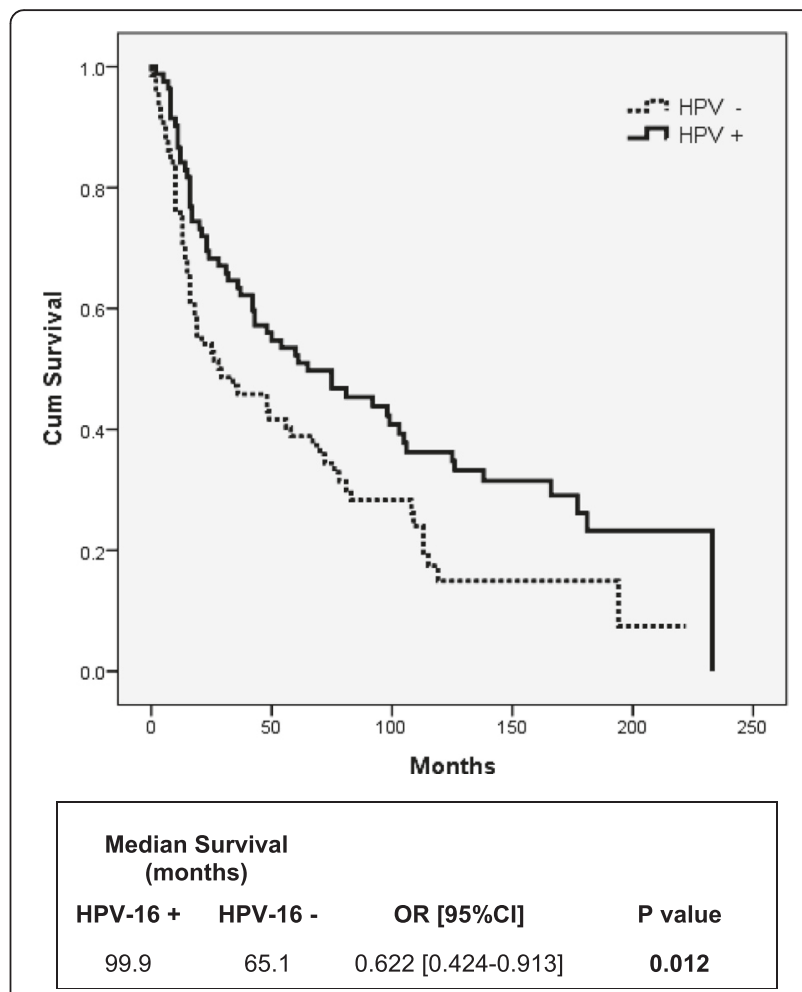

Fig. 6 Overall survival of HNSCC patients with heavy alcohol consumption history according to HPV-16 status. Kaplan-Meier survival curve analysis of HPV-16 positive versus HPV-16 negative HNSCC patients with a history of heavy alcohol consumption

radiotherapy when compared to HPV-16 negative patients. This increase in radiosensitivity could be mediated through wild-type p53-mediated apoptosis in HPV-16 positive cells and a lower chromosomal loss [42].

The primary limitations of this study are the small number of early stages (I and II) HNSCC samples, and a small group of PSCC's in our study cohort. These limitations may explain why we did not observe a statistical difference in early stage tumors by HPV-16 status. Our HNSCC patient population is composed mostly of late stage tumors (III, IV), predominantly from LSCC and OSCC. This issue occurs because our head and neck cancer service is attached to a supraterciary level hospital care center which is responsible for the management of complex cancer cases for the whole island of Puerto Rico.

\section{Conclusions}

In this study we showed that, HPV-16 presence, in HNSCC tumors, causes an increase in OS and increases radiosensitivity of tumor cells. Interestingly, we have shown that HPV-16 is present, not only in OPSCC as previously described, but it was also detected in LSCC and OSCC. Although the TNM classification has been effective for prognostication of HNSCC, HPV-16 
detection may serve as a potential biomarker, in combination with the TNM, to better establish the prognosis of LSCC and PSCC and guide treatment. Future work should be directed to understand how HPV-16 affects HNSCC carcinogenesis, and how its infection modifies the disease progression increasing the OS in these patients.

\section{Abbreviations}

HNSCC, head and neck squamous cell carcinoma; HPV, human papillomavirus; IHC, immunohistochemistry; LSCC, larynx squamous cell carcinoma; OPSCC, oropharynx squamous cell carcinoma; OS, overall survival; OSCC, oral cavity squamous cell carcinoma; PSCC, pharynx squamous cell carcinoma; qPCR, quantitative real-time polymerase chain reaction; RTX, radiotherapy; TNM, TNM classification of malignant tumors

\section{Acknowledgements}

The research described was supported by the University of Puerto Rico School of Medicine Otolaryngology Section, NIH/National Cancer Institute grants P20CA91402, U54CA96297; NIH/National Institute of General Medical Sciences grant S06GM8224. This research also used core facilities supported by NIH/NCRR G12RR03051 and NIH/NIMHDD 8G12MD007600. Additionally, we want to thank Antonio Arrieta, MSII, for the assistance on the statistical part of this study.

\section{Availability of data and materials}

The authors are cognizant and agree to abide to NIH data sharing policies. Raw data from this study will be made accessible only to those who agree to defined conditions of use, and to individuals who meet professional criteria as appropriate

\section{Authors' contributions}

$B R, F R$, JT \& AB performed the study design. FR, JT \& AB consented HNSCC patients to the study and collected all HNSCC tumor samples. MM carried out the histopathological analysis of the samples. BR \& GV carried out the DNA extraction and HPV screening using qPCR on all samples. RR, BR \& FR performed the statistical analysis. All authors drafted, revised and approved the final manuscript.

\section{Competing interests}

The authors declare that they have no competing interests with the presented study.

\section{Ethics approval and consent to participate}

All study procedures have the approval of the University of Puerto Rico-Medical Sciences Campus IRB (MSC-IRB Protocol 2770103). Informed consent was obtained from all study participants.

\section{Author details}

'Department of Biology, Natural Sciences Faculty, University of Puerto Rico, Rio Piedras Campus, San Juan, Puerto Rico. Department of Otolaryngology, Head and Neck Surgery Section, University of Puerto Rico, School of Medicine, PO Box 365067, San Juan 00936, Puerto Rico. ${ }^{3}$ Department of General Social Sciences, University of Puerto Rico, Rio Piedras Campus, San Juan, Puerto Rico. ${ }^{4}$ Department of Pathology and Laboratory Medicine, University of Puerto Rico, School of Medicine, San Juan, Puerto Rico. ${ }^{5}$ Medical Services Administration, San Juan, Puerto Rico. ${ }^{6}$ Department of Pharmacology and Toxicology, University of Puerto Rico, School of Medicine, San Juan, Puerto Rico.

\section{Received: 14 May 2016 Accepted: 29 July 2016}

Published online: 24 August 2016

\section{References}

1. Ferlay J, Shin HR, Bray F, Forman D, Mathers C, Parkin DM. Estimates of worldwide burden of cancer in 2008: GLOBOCAN 2008. Int J Cancer. 2010; 127(12):2893-917.

2. O'Rorke MA, Ellison MV, Murray LJ, Moran M, James J, Anderson LA. Human papillomavirus related head and neck cancer survival: A systematic review and meta-analysis. Oral Oncol. 2012;48(12):1191-201.
3. American Medical Association. ICD-10-CM 2016: The Complete Officia Codebook. 2016 ed. Chicago: American Medical Association; 2016.

4. Shiboski CH, Schmidt BL, Jordan RC. Tongue and tonsil carcinoma: increasing trends in the US population ages 20-44 years. Cancer. 2005; 103(9):1843-49.

5. Báez A. Genetic and environmental factors in head and neck cancer genesis. J Environ Sci Health C Environ Carcinog Ecotoxicol Rev. 2008;26(2):174-200.

6. Pulte $\mathrm{D}$, Brenner $\mathrm{H}$. Changes in Survival in Head and Neck Cancers in the Late 20th and Early 21st Century: A Period Analysis. Oncologist. 2010;15(9): 994-1001.

7. Figueroa NR, Ortiz KJ, Pérez N, Villanueva E, Traverso M, Torres CR, et al. Cancer in Puerto Rico, 2004-2009. San Juan: Puerto Rico Central Cancer Registry; 2012

8. Blot WJ, McLaughlin JK, Winn DM, Austin DF, Greenberg RS, Preston-Martin $\mathrm{S}$, et al. Smoking and drinking in relation to oral and pharyngeal cancer. Cancer Res. 1988;48(11):3282-7.

9. Saracci R. The interactions of tobacco smoking and other agents in cancer etiology. Epidemiol Rev. 1987;9:175-93.

10. Hashibe M, Brennan P, Benhamou S, Castellsague X, Chen C, Curado MP, et al. Alcohol drinking in never users of tobacco, cigarette smoking in never drinkers, and the risk of head and neck cancer: pooled analysis in the International Head and Neck Cancer Epidemiology Consortium. J Natl Cancer Inst. 2007:99(10):777-89.

11. Gillison ML, Koch WM, Capone RB, Spafford M, Westra WH, Wu L, et al. Evidence for a causal association between human papillomavirus and a subset of head and neck cancers. J Natl Cancer Inst. 2000;92(9):709-20.

12. Mork J, Lie AK, Glattre E, Hallmans G, Jellum E, Koskela P, et al. Human papillomavirus infection as a risk factor for squamous-cell carcinoma of the head and neck. N Engl J Med. 2001;344(15):1125-31.

13. Báez A, Almodóvar JI, Cantor A, Celestin F, Cruz-Cruz L, Fonseca S, et al. High frequency of HPV16-associated head and neck squamous cell carcinoma in the Puerto Rican population. Head Neck. 2004;26(9):778-84.

14. IARC Monograph. Human Papillomaviruses. IARC. 2012;100B:255-313.

15. Van Doorslaer K, Tan Q, Xirasagar S, Bandaru S, Gopalan V, Mohamoud Y, et al. The Papillomavirus Episteme: a central resource for papillomavirus sequence data and analysis. Nucleic Acids Res. 2013:41:D571-8.

16. Scheffner M, Werness BA, Huibregtse JM, Levine AJ, Howley PM. The E6 oncoprotein encoded by human papillomavirus types 16 and 18 promotes the degradation of p53. Cell. 1990;63(6):1129-36.

17. Dyson N, Howley PM, Münger K, Harlow E. The human papilloma virus-16 E7 oncoprotein is able to bind to the retinoblastoma gene product. Science. 1989;243(4893):934-37.

18. Gillison ML, Shah KV. Human papillomavirus-associated head and neck squamous cell carcinoma: Mounting evidence for an etiologic role for human papillomavirus in a subset of head and neck cancers. Curr Opin Oncol. 2001;13(3):183-88

19. Fakhry C, Zhang Q, Nguyen-Tan PF, Rosenthal D, El-Naggar A, Garden AS, et al. Human papillomavirus and overall survival after progression of oropharyngeal squamous cell carcinoma. J Clin Oncol. 2014:32(30):3365-73.

20. Gillison ML, D'Souza G, Westra W, Sugar E, Xiao W, Begum S, et al. Distinct risk factor profiles for human papillomavirus type 16-positive and human papillomavirus type 16-negative head and neck cancers. J Natl Cancer Inst. 2008:100(6):407-20.

21. Deschler DG, Richmon JD, Khariwala SS, Ferris RL, Wang MB. The "new" head and neck cancer patient-young, nonsmoker, nondrinker, and HPV positive: evaluation. Otolaryngol Head Neck Surg. 2014;151(3):375-80.

22. Goldenberg D, Begum S, Westra WH, Khan Z, Sciubba J, Pai SI, et al. Cystic lymph node metastasis in patients with head and neck cancer: An HPVassociated phenomenon. Head Neck. 2008;30:898-903.

23. Schwartz SR, Yueh B, McDougall JK, Daling JR, Schwartz SM. Human papillomavirus infection and survival in oral squamous cell cancer: a population-based study. Otolaryngol Head Neck Surg. 2001;125(1):1-9.

24. Dayyani F, Etzel CJ, Liu M, Ho CH, Lippman SM, Tsao AS. Meta-analysis of the impact of human papillomavirus (HPV) on cancer risk and overall survival in head and neck squamous cell carcinomas (HNSCC). Head Neck Oncol. 2010:2:2-15.

25. Stadler ME, Patel MR, Couch ME, Hayes DN. Molecular biology of head and neck cancer: Risks and pathways. Hematol Oncol Clin North Am. 2008;22(6): 1099-124.

26. American Joint Committee on Cancer. AJCC Cancer Staging Handbook 7. 7th ed. New York: Springer; 2011. 
27. de Roda Husman AM, Walboomers JM, van den Brule AJ, Meijer CJ, Snijders PJ. The use of general primers GP5 and GP6 elongated at their 3' ends with adjacent highly conserved sequences improves human papillomavirus detection by PCR. J Gen Virol. 1995;76(4):1057-62.

28. Báez A, Cantor A, Fonseca S, Marcos-Martinez M, Mathews LA, Muro-Cacho CA, et al. Differences in Smad4 expression in human papillomavirus type 16positive and human papillomavirus type 16-negative head and neck squamous cell carcinoma. Clin Cancer Res. 2005;11(9):3191-7.

29. Fonseca S. The Incidence, Prognostic and Therapeutic Significance of HPV16 E6/E7 Oncoproteins in Head and Neck Squamous Cell Carcinoma in Puerto Ricans. [master's thesis]. San Juan: University of Puerto Rico - School of Medicine; 2006. p. 67.

30. Bol V, Grégoire V. Biological basis for increased sensitivity to radiation therapy in HPV-positive head and neck cancers. Biomed Res Int. 2014;2014:1-6.

31. Fakhry C, Westra WH, Li S, Cmelak A, Ridge JA, Pinto H, et al. Improved Survival of Patients With Human Papillomavirus - Positive Head and Neck Squamous Cell Carcinoma in a Prospective Clinical Trial. J Natl Cancer Inst. 2008;100(4):261-69.

32. Annertz $K$, Rosenquist $K$, Andersson $G$, Jacobsson $H$, Hansson BG, Wennerberg J. High-risk HPV and survival in patients with oral and oropharyngeal squamous cell carcinoma - 5-year follow up of a population-based study. Acta Otolaryngol. 2014;134(8):843-51.

33. Nagel R, Martens-de Kemp SR, Buijze M, Jacobs G, Braakhuis BJ, Brakenhoff $\mathrm{RH}$. Treatment response of HPV-positive and HPV-negative head and neck squamous cell carcinoma cell lines. Oral Oncol. 2013;49(6):560-66.

34. Smeets SJ, Braakhuis BJ, Abbas S, Snijders PJ, Ylstra B, van de Wiel MA, et al. Genome-wide DNA copy number alterations in head and neck squamous cell carcinomas with or without oncogene-expressing human papillomavirus. Oncogene. 2006;25(17):2558-64.

35. Sepiashvili L, Bruce JP, Huang SH, O'Sullivan B, Liu FF, Kislinger T. Novel insights into head and neck cancer using next-generation "omic" technologies. Cancer Res. 2015;75(3):480-86.

36. Perez-Ordonez B, Beauchemin M, Jordan RC. Molecular biology of squamous cell carcinoma of the head and neck. J Clin Pathol. 2006;59(5):445-53.

37. Braakhuis BJ, Snijders PJ, Keune WJ, Meijer C, Ruijter-Schippers HJ, Leemans $C$, et al. Genetic patterns in head and neck cancers that contain or lack transcriptionally active human papillomavirus. J Natl Cancer Inst. 2004; 96(13):998-1006

38. Ramshankar V, Krishnamurthy A. Human papilloma virus in head and neck cancers-role and relevance in clinical management. Indian J Surg Oncol. 2013;4(1):59-66.

39. Ang KK, Harris J, Wheeler R, Weber R, Rosenthal DI, Nguyen-Tân PF, et al. Human papillomavirus and survival of patients with oropharyngeal cancer. N Engl J Med. 2010;363(1):24-35.

40. Rieckmann T, Tribius S, Grob TJ, Meyer F, Busch CJ, Petersen C, et al. HNSCC cell lines positive for HPV and p16 possess higher cellular radiosensitivity due to an impaired DSB repair capacity. Radiother Oncol. 2013;107(2):242-46.

41. Smith EM, Rubenstein LM, Haugen TH, Pawlita M, Turek LP. Complex etiology underlies risk and survival in head and neck cancer human papillomavirus, tobacco, and alcohol: a case for multifactor disease. J Oncol. 2012;2012(57):1-9.

42. Young RJ, Rischin D, Fisher R, McArthur GA, Fox SB, Peters $\amalg$, et al. Relationship between epidermal growth factor receptor status, p16(INK4A), and outcome in head and neck squamous cell carcinoma. Cancer Epidemiol Biomarkers Prev. 2011;20(6):1230-37.

\section{Submit your next manuscript to BioMed Central and we will help you at every step:}

- We accept pre-submission inquiries

- Our selector tool helps you to find the most relevant journal

- We provide round the clock customer support

- Convenient online submission

- Thorough peer review

- Inclusion in PubMed and all major indexing services

- Maximum visibility for your research

Submit your manuscript at www.biomedcentral.com/submit
Biomed Central 